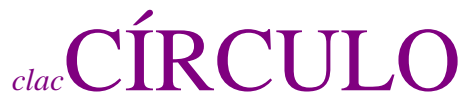 \\ de
lingüística \\ lingüistica
aplicada a la \\ comunica
}

$62 / 2015$

\section{VALORES DISCURSIVOS DE PORQUE EN CONSTRUCCIONES CAUSALES TAUTOLÓGICAS}

\author{
Rosario González Pérez \\ Universidad Autónoma de Madrid \\ rosario gonzalez en uam es
}

Resumen

El presente artículo tiene como objetivo el análisis de los valores discursivos de porque en construcciones tautológicas, que suponen, por tanto, la repetición del primer miembro: Va a llorar, porque va a llorar / No lo sé porque no lo sé. En estas construcciones el sentido causal está tan debilitado que llega a desaparecer. Tras un acercamiento al fenómeno de la causalidad, se describen las características y tipos de las construcciones causales tautológicas y se ofrece una explicación discursivo-pragmática: las causales tautológicas evidenciales ( Va a llorar, porque va a llorar), marcan la seguridad de lo afirmado, apuntando a la fuente de la información transmitida, explícita o implícita en los conocimientos, creencias o expectativas del hablante; las causales tautológicas de bloqueo de la réplica (No lo sé porque no lo sé) se convierten en una estrategia para evitar un turno reactivo y pueden constituir un tipo de explicatura y dar lugar a procesos de desambiguación para suprimir la posibilidad de interpretación como causal pura.

Palabras clave: discurso, causalidad, porque, construcciones tautológicas

González Pérez, Rosario. 2015..

Valores discursivos de porque en construcciones causales tautológicas.

Círculo de Lingüística Aplicada a la Comunicación 62, 32-53.

http://www.ucm.es/info/circulo/no62/gonzalezP.pdf

http://revistas.ucm.es/index.php/CLAC

http://dx.doi.org/10.5209/rev_CLAC.2015.v62.49497

(C) 2015 Rosario González Pérez

Círculo de Lingüística Aplicada a la Comunicación (clac)

Universidad Complutense de Madrid. ISSN 1576-4737. http://www.ucm.es/info/circulo 


\begin{abstract}
Discourse values of Spanish porque (because) in causal tautologies

This article aims to analyse the discursive values of tautological constructions with porque that imply, therefore, repetition of the first member: Va a llorar, porque va a llorar / No lo sé porque no lo sé. In these constructions the causal sense is so weakened that it eventually disappears. Based on a theoretical approach to the question of causation, characteristics and types of causal tautological constructions are described and their discursive and pragmatic implications are explained: the evidential tautological causal sentences (Va a llorar, porque va a llorar) mark the certainty of the assertion, pointing to the source of the transmitted information, expressed or implied in the knowledge, beliefs, and expectations of the speaker; tautological causal sentences used to block the reply (No lo sé porque no lo sé) become a strategy to avoid a reactive turn and can be a type of explicature and lead to disambiguation processes to remove the possibility of interpretation of this kind of sentences as pure causal.
\end{abstract}

Key words: discourse, cause, porque, tautological constructions

Índice

Resumen 32

Abstract 33

1. Introducción 34

2. La conexión causal y porque 36

3. Causalidad y modalidad epistémica: las causales tautológicas 40

3.1. Las causales tautológicas y el bloqueo de la réplica 45

3.2. Los marcadores de evidencialidad y porque 47

4. Conclusiones 49

Bibliografía 51 


\section{Introducción}

Es bien sabido que numerosos nexos oracionales adoptan usos discursivo-pragmáticos que modifican sus valores funcionales y semánticos prototípicos ${ }^{1}$. En el ámbito semántico de la causalidad que, según Galán Rodríguez (1999: 3599) “abarca cinco relaciones diferentes (causales propiamente dichas, finales, condicionales [...], concesivas [...] y consecutivas [...]) que se organizan según la particular concepción que en cada una de ellas se establece entre la causa y el efecto [...]", encontramos casos como los que siguen:

(1) Una pérdida de balón de Parejo, fue aprovechada por Diego, quien centró un balón para que Adrián controlase a la perfección con el pecho y cruzase el balón con un potente disparo a la escuadra lejos del alcance de Alves. (Público.es, Deportes, 27 de abril de 2012) $)^{2}$

(2) Si la empresa ya era difícil para el Valencia, en ese momento se convirtió prácticamente en imposible. (Público.es, Deportes, 27 de abril de 2012)

(3) Va a llorar, porque va a llorar ${ }^{3}$. (Conversación [G.68.B.J + G.69.A.J], Grupo Val.Es.Co 1995: 201)

En el ejemplo (1) observamos cómo en el nexo para que, prototípico para la expresión de la finalidad, queda desdibujada la relación causa-efecto característica de las oraciones finales, que sí puede observarse en la oración final canónica:

(4) Lo he traído para que lo veas (donde q: para que lo veas se interpreta como posterior a $\mathrm{p}$ : lo he traído)

En el ejemplo (1) es dudoso interpretar como relación causa-efecto, el efecto 'Adrián controló el balón con el pecho', provocado por la causa 'Diego centró un balón', en tanto que percibimos sin problemas la secuenciación cronológica de las dos acciones. Y

\footnotetext{
${ }^{1}$ Este trabajo es resultado del Proyecto de investigación Semántica latino-románica: unidades de significado procedimental. Diferencias conservadoras e innovadoras del latín al español y afinidades con otras lenguas (referencia FFI2012-34826, 2012-2015) del Ministerio de Economía y Competitividad. Este proyecto se desarrolla bajo la dirección del IP Benjamín García Hernández.

${ }^{2}$ Este tipo de ejemplos con para que predominan en los medios periodísticos, lo que ya ha sido señalado por varios autores (Herrero Ruiz de Loizaga 2005: 382).

${ }^{3}$ En este caso el contexto del ejemplo es una conversación coloquial prototípica en que el hablante $\mathrm{P}$, mujer, de más de 55 años y baja formación, reproduce las palabras de otro al relatar una situación anterior, es decir, trae a la conversación un discurso referido. Se trata de la conversación entre el cirujano que va a operar a un niño (intervención quirúrgica menor) y su madre, conversación a la que asiste $\mathrm{P}$ y que, según la transcripción del grupo Val.Es.Co, P está reproduciendo en el curso de una conversación posterior. P formula el enunciado lingüístico con sus propias palabras, no habla como presumiblemente lo haríael cirujano y en este contexto dice lo que sigue:

P: [...] /total que/ el otro niño vino $\uparrow /$ le tomaron la temperatura $\uparrow$ tenía fiebre y no le pudieron operar/ así que pasó él el primero// pasó él y nos dijo el cirujano/ no os asustéis/ va a llorar// porque va a llorar $\downarrow$ - cuan - cuando se lo llevaron $\uparrow$ no/ porque empezaron a gastarle bromas $\uparrow /$ (Conversación [G.68.B.J+G.69.A.J], Grupo Val.Es.Co 1995: 201)
} 
claramente queda suspendida, en favor de la secuenciación temporal, la relación causaefecto propia de la finalidad en

(5) Felipe centra a Juanito para que éste falle una clara oportunidad. (Herrero Ruiz de Loizaga 2005: 382)

En (2) sucede lo mismo con el condicional si: el esquema [si $p, q]$, en el que suponemos que si se da la premisa $p$, llevará al efecto $q$, como en

(6) Si mañana hace sol, iremos a la playa (Montolío 1999: 3648)

no puede cumplirse en (2), con una oración en pasado, de carácter asertivo y con la prótasis en indicativo en que percibimos, como en (1), la secuenciación cronológica y la intensificación en $q$ 'ahora es imposible' de lo expresado en $p$ 'antes la empresa era difícil'. Como en el caso de la finalidad, la temporalidad es un elemento relevante en las construcciones condicionales orientadas hacia el futuro (Montolío 1999: 3726-3728), lo que permite la contraposición de dos acciones secuenciadas cronológicamente.

Por su parte en (3) porque marca la seguridad de lo afirmado, apuntando a la fuente de la información transmitida, ya sea esta explícita o esté implícita en los conocimientos, creencias o expectativas del hablante. Así, pasa a expresar la intensificación e incluso la confirmación fuerte; como se desprende de la repetición, tras pausa marcada con tonema en cadencia, del primer argumento asertivo, pero esta vez introducido mediante porque. A partir de aquí, porque pasa también a indicar el compromiso explícito y patente del hablante con la información expresada e inferida, y se sitúa en la órbita de marcadores de modalidad epístémica evidenciales como desde luego, por supuesto o el conversacional claro. En este caso, lo interesante de la construcción radica en la escasa atención de este uso de porque.

Mientras que diversos autores se han ocupado de ejemplos como los de (1) (para que) o (2) (si), ofreciendo explicaciones para estos empleos o, al menos, señalando su existencia. En el caso del valor evidencial (y, por eso también confirmativo fuerte) de porque, no existen, que sepamos, referencias a este uso. Es muy posible que en ello haya influido el carácter marcadamente conversacional del empleo, lo que hace muy difícil su rastreo. En

\footnotetext{
${ }^{4}$ Este autor señala que estos casos se explican como "construcciones de carácter cronológico en las que la oración introducida por para (que) indica una acción posterior a la de la oración principal. [...] El hecho de que la finalidad implique una proyección hacia el futuro, y por tanto posterioridad respecto de la oración principal puede facilitar este desplazamiento hacia lo temporal.” (Herrero Ruiz de Loizaga 2005: 380).
} 
los dos volúmenes que transcriben conversaciones coloquiales, publicados por el grupo Val.Es.Co., solamente hemos encontrado un empleo de porque con este valor (3). Buscar en corpus de referencia es también problemático, dado que porque es el elemento prototípico para establecer la conexión causal en español con todos sus matices ${ }^{5}$, lo que supone una frecuencia de aparición inabarcable en la práctica. Por ello, en este trabajo de toma de contacto con el fenómeno estudiado, partimos de una serie de ejemplos en que, como puede observarse, el fenómeno estudiado es recurrente, lo que nos sirve para plantear el problema, aplicar una hipótesis, y establecer un análisis pragmático-discursivo del fenómeno. Además, resulta de interés analizar entornos discursivos en los que elementos de contenido lingüístico codificado queden habilitados, aunque sea en contextos muy específicos, para la expresión de valores pragmáticos con anulación del contenido codificado, porque este tipo de análisis puede arrojar luz sobre las condiciones que favorecen (extensión a otros contextos, pérdida de requisitos formales, ambigüedad en la interpretación de la ocurrencia, pues no se puede discriminar entre lo dicho, lo explicado y lo implicado, desplazamiento o supresión de los usos codificados)el paso del entorno contextual específico a la fijación del significado procedimental ${ }^{6}$.

\section{La conexión causal y porque}

La distinción latina entre 'causa real' (la que expresa el fundamento de una acción) y 'causa lógica' (la que justifica una opinión o un juicio sabido de antemano) está en la base de la distinción tradicional de las causales. Debemos a Bello la atinada precisión operada sobre los dos tipos de causa, por un lado la causa de lo dicho, que se correspondería con la

\footnotetext{
${ }^{5}$ En otras lenguas la situación para la expresión de la conexión causal es diferente. En francés, por ejemplo, los nexos tipo son tres: parce que, car y puisque. Los tres se reparten los distintos valores causales y los distintos registros, oral y escrito. Parce que, aparece en lo oral y en lo escrito y asume tanto la expresión de la causa objetiva como la introducción de otro tipo de argumentos causales (justificación, deducción a partir de indicios, etc.). Car, prácticamente ha desaparecido del registro oral en francés moderno y se especializa en la expresión de argumentos más que de causas reales. Puisque, por su parte, es el único conector causal que puede introducir un miembro discursivo que no esté conectado a otro anterior. El caso del because inglés es bastante parecido aldel porque en español. No hay en las dos lenguas una especialización entre conectores causales de la enunciación y del enunciado, como en francés.

${ }^{6}$ Para poder hacer un estudio de este tipo sería interesante rastrear el fenómeno estudiado (al menos en cortes cronológicos)y aplicar la metodología de la lingüística del corpus, que nos permitiría basarel análisis cualitativo en un análisis cuantitativo previo y obtener datos objetivosque pueden validarse o no con nuevos datos. Pero para ello habría quecontar con un corpus sobre porque del que por el momento no disponemos. Los ejemplos que se ofrecen a lo largo del trabajo han sido recogidos durante los años 2012 y 2013, a partir del visionado y escucha de programas de televisión y radio (noticiarios, series que recrean la oralidad) y de la escucha de conversaciones prototípicas espontáneas.
} 
causa real y, por otro, la causa del decir, que se correspondería con la causa lógica, de modo que el gramático venezolano opera un salto cualitativo al situar las causas en el terreno de lo lingüístico (Bello [1847] 1998: vol. II, § 991d, 618) ${ }^{7}$. En la tradición hispánica, las construcciones causales denominadas 'causales del enunciado' y 'causales de la enunciación' recogen los dos tipos de causa propuestos por Bello ${ }^{8}$. Por su parte, Galán Rodríguez (1999: 3602) ${ }^{9}$ establece una clasificación semántica en dos tipos a los que llama 'causales puras' (causales propiamente dichas, en las que la relación entre las oraciones es no consabida y se manifiesta a través de una relación de causa-efecto o de motivo-resultado)

(7a) Las flores se han marchitado porque no tenían agua suficiente (causa-efecto)

(7b) Te he comprado un regalo porque era tu cumpleaños (motivo-resultado)

y 'causales explicativas' (una oración expresa un hecho B que se presenta como explicación razonable o como justificación de la oración A); a su vez las causales explicativas pueden subdividirse en 'causales explicativas propias' (presentan circunstancias favorables o habituales para explicar una acción) y en 'causales hipotéticas' (presentan deducciones a partir de unos hechos que nos sirven de base para explicar o justificar lo dicho en otra oración).

(8a) Ha llovido, porque el suelo está mojado (causal explicativa propia)

(8b) No se ha ido, porque tiene ahí la cartera (causal hipotética ${ }^{10}$ )

Existe, además, una relación diferente entre el contenido proposicional de las dos oraciones en conexión causal: en las causales puras el grado de dependencia del predicado con respecto al antecedente es mucho mayor que en las causales explicativas, de hecho, las causales puras están integradas en el predicado verbal y cuando van pospuestas, lo que

\footnotetext{
${ }^{7}$ Bello lo expresa de este modo: "Así, en «Huyó porque le acometieron muchos a un tiempo», la huida es el efecto de la acometida." ([1847] 1998: 619). En otros casos, sin embargo, expresa "el fundamento que hemos tenido para enunciar la primera [proposición].” ([1847] 1998: 619).

${ }^{8}$ Marcos Marín (1979) propone la denominación 'causales del enunciado' / 'causales de la enunciación', que tanta fortuna ha tenido en la literatura especializada al respecto. Con anterioridad, Lapesa (1978) se refería a las dos construcciones causales como 'causales de la acción enunciada' y 'causales del acto enunciativo'. Para una información más detallada sobre esta dicotomía y su denominación v. Galán Rodríguez (1999: 3601, nota 7).

${ }^{9}$ Sigo la propuesta de clasificación de esta autora -aunque solo en parte la terminología que usa- ,que puede
consultarse en Galán Rodríguez (1999: 3602). Los ejemplos (7a), (7b) y (8b) también proceden de ella.
${ }^{10}$ Esta terminología puede llevar a equívocos. En primer lugar, la hipótesis está en elprimer miembro del
enunciado; en segundo lugar, existen otros esquemas denominados causales hipotéticosdel tipo: Lo hice por
si venía. Para las causales explicativas indiciales o justificativas, preferimos la denominación, tomada de
Sweetser (1990) ‘causales epistémicas'.
} 
constituye su orden canónico, no hay pausa entre antecedente y consecuente, lo que denota la existencia de un único acto de habla. Son lo que se denomina 'causales integradas o centrales'. En contraposición a ellas, las causales explicativas, manifiestan una relación menos estrecha con el predicado verbal del antecedente; lo que podemos comprobar en la presencia de una pausa entre antecedente y consecuente que separa dos actos de habla diferenciados: uno como aserción y otro como explicación o justificación de lo afirmado en el acto precedente. Esta relativa separación formal entre antecedente y consecuente es lo que hace que nos podamos referir a ellas como 'causales periféricas'"11.

Pues bien, esta excesiva simplificación tipológica con la que acabamos de esbozar las relaciones causales, no implica que no existan muy variados matices en la forma de presentar la causa por parte del hablante. Esos matices se ordenan de forma gradual desde la causa real hasta la pérdida del valor causal prototípico, como hemos visto en (3), pasando por otros múltiples sentidos que se acumulan, sobre todo, en la zona significativa de las causales explicativas ${ }^{12}$. Son estas diferencias semánticas y pragmáticas entre los distintos tipos de conexión causal las responsables de las posibilidades combinatorias y del comportamiento formal de los distintos nexos (porque, como, puesto que, ya que, visto que, supuesto que, etc.) en relación con la negación, la interrogación, el orden de las secuencias y la alternancia con otros nexos o construcciones para la expresión de la causalidad.

La idea de una organización gradual, que va de los valores objetivos a los valores subjetivos en la expresión de la causalidad, para interpretar los usos semánticopragmáticos de la conexión causal y su nexos es relativamente reciente. Así lo señala

\footnotetext{
${ }^{11} \mathrm{La}$ diferencia entre ambos tipos, las causales integradas y las periféricas, se aprecia con claridad en los siguientes ejemplos. En ellos, la pausa es la responsable de la interpretación como causal integrada en a) y causal periférica en b)

a) He escrito a Juan porque se lo heprometido.

b) Escribe a Juan, porque se lo has prometido. (Galán Rodríguez 1999: 3609)

${ }^{12}$ Basta con consultar el Diccionario de partículas de Santos Ríos (2003), para comprobar la extensión de estos matices discursivo-pragmáticos a los que están dedicadas las páginas 518 a la 523. Sin embargo, como es habitual en las clasificaciones sobre marcadores, que suelen excluir las conjunciones, porque no se incluye en el Diccionario de conectores y operadores del español(Fuentes Rodríguez 2009). Tampoco se incluye entre la selección de las partículas del DPDE (Diccionario de partículas discursivas del español); Briz señala la razón de la exclusión cuando dice"En la selección de las partículas del DPDE [...] no se han incluido las formas conjuntivas (pero, y, si, o, porque, aunque), ni la mayoría de los adverbios en -mente, entre otras razones, porque solo son partículas ocasionalmente y, además, porque los criterios para establecer dicha funcionalidad no son siempre seguros: en el caso de las conjunciones, al estar más vinculadas o integradas en la proposición”.(Briz 2011: 86)
} 
Zufferey (2012: 24), en su estudio pragmático sobre la contraposición de los nexos car, parce que y puisque en francés ${ }^{13}$, que explica a partir de la clasificación tripartita de Sweetser (1990) para because, en la que Sweetser distingue tres valores principales: 'content or real word uses'.

(9a) He fell because I pushed him(en que el hablante presenta como inapelable lo expresado en $q$ )

'speech act uses'

(9b) Are you ready? Because we are late(en que existen dos actos de habla separados y $q$ se presenta como justificación de la pregunta $p$ )

Y 'epistemic uses'

(9c) Heisaway, becausethelights are out(en que el hablante presentaunaopinión, creencia o conclusión a la que ha llegado a partir de unos indicios; se deduce $p$ a partir de $q$ )

Lo interesante del análisis de Zufferey es la interpretación de algunos usos (epistémicos) de puisque ${ }^{14}$, como empleos del valor ecoico del lenguaje en el sentido en que lo usan Sperber y Wilson (1986, 1994: 290-295; Wilson y Sperber 2004:607-632), con o sin intención irónica. Estos autores, partiendo del concepto de literalidad interpretativa distinguen entre uso y mención ecoica. En la mención ecoica el enunciado ecoizado pertenece al cotexto y es repetición de un enunciado previo (Sperber y Wilson 1986). Pero muchas veces, como sucede en el caso de las causales evidenciales tautológicas, el enunciado ecoico no se encuentra en enunciados inmediatos, sino en contextos anteriores que activan supuestos contextuales de carácter histórico, cultural, experiencial, etc. Estos supuestos contextuales pertenecen al entorno cognitivo y se alojan en la memoria a largo y corto plazo. Por eso se activan cuando no se puede identificar exactamente la fuente del eco. La concepción más amplia de los usos ecoicos del lenguaje, está en relación con el uso descriptivo e interpretativo del lenguaje y, en este segundo caso (el empleo

\footnotetext{
${ }^{13}$ Señala Zufferey (2012: 24)que "Morerecently, some authors like Pander Maat and Degand (2001) or Pit (2003) had advocated a more fine-grained division, based in a scalar notion going from objective (content) to subjective (speech act) relations."

${ }^{14}$ Distinta, aunque muy relacionada,es la interpretación de Wüest (2012) para la contraposición parce que, car y puisque. Superando en el análisis de estos tres conectores la dicotomía del Groupe $\lambda$-1 (logique et langage) (1975), que distingue entre usos semánticos como operador para parce quey usos como marcadores de actos de habla para car y puisque, Wüest (2012: 91-93), aplica la teoría de los esquemas argumentativos denominados topoï (sobre todo el intrínseco) a su análisis, lo que le lleva a considerar puisque como el prototipo de conector argumentativo para la introducción de argumentos ya conocidos o incluso sobreetendidos. Esta interpretación acerca su visión pragmática de puisque, aunque por otra vía, a la de Zufferey.
} 
interpretativo), con dos tipos de empleo interpretativo: el marcado explícitamente y el uso tácito (Wilson y Sperber 2004: 607-632). Por decirlo de otro modo, estos usos activan efectos contextuales que quedan implícitos en el discurso por consabidos, ya por haber sido explícitos en una enunciación anterior o por formar parte de los supuestos compartidos. De este modo, la relevancia se obtiene de la evaluación del enunciado mismo en relación con la situación de emisión. La tautología sería el caso extremo de mención ecoica inmediata, pero no es la voz de otros enunciadores la que se trae al discurso, como en la ironía, sino la del propio interlocutor. De ahí que la imposibilidad de asignar un contenido proposicional literal a la construcción emitida nos lleve a usar el lenguaje de forma interpretativa y a buscar la interpretación más accesible en el entorno cognitivo: 'si alguien dice esto como lo dice es porque tiene razones fundadas para hacerlo, es decir, está autorizado a hacerlo, ${ }^{15}$. Así las construcciones evidenciales con porque (ejemplo 3) nos permiten señalar lo evidente de lo afirmado, dar esta información como consabida, por tanto, sin necesidad de formulación explícita y presentar lo que en realidad es opinión propia (valor epistémico de porque) como indiscutible (valor de confirmación fuerte de porque $)^{16}$.

\section{Causalidad y modalidad epistémica: las causales tautológicas}

La modalidad epistémica es aquella por la cual el hablante presenta en sus enunciados sus creencias, conjeturas u opiniones. Las causales explicativas y dentro de ellas, especialmente las epistémicas, pertenecen a esta modalidad. Los usos de porque que

\footnotetext{
${ }^{15}$ Pensemos en las condiciones preparatorias de los actos de habla.

${ }^{16}$ El uso literal del lenguaje sería el punto de partida de Grice ([1975] 1991) para la explicación de estas construcciones. Para este autor,el principio de cooperación ("haga su contribución al mensaje tal y como lo exija el punto del intercambio comunicativo en que se encuentre") regula la comunicación humana y se concreta a partir de una serie de máximas o instrucciones cuyo cumplimiento se da por descontado en la comunicación humana. Cuando un hablante es aparentemente no cooperativo en un intercambio, ponemos en relación lo dicho con el contexto en que lo emitimos y, dando por sentado que el principio de cooperación regula los intercambios, buscamos el significado intencional del enunciado emitido, es decir, inferimos lo comunicado. Esta podría ser una explicación para las causales evidenciales, pero no podría explicar por qué son posibles tanto la interpretación literal como la no literal en otro tipo de causales tautológicas que, más que un tipo diferenciado, constituyen una estrategia de bloqueo de la réplica y a las que nos referiremos más adelante (No lo sé porque no lo sé, ejemplo 28). En este caso, inferimos desde las posibilidades de codificación del enunciado proposicional en relación con el contexto de emisión. Necesitamos de un enriquecimiento pragmático del enunciado emitido que en el ejemplo 28 supone un proceso de desambiguación que correspondería al nivel de lo explicado de Sperber y Wilson. Una postura más abarcadora que la de Grice (Carston 2012, especialmente las págs. 471-478) permite explicar ambos tipos de interpretaciones causales tautológicas, las evidenciales y las de bloqueo de la réplica.
} 
vamos a analizar también constituyen usos epistémicos. Retomemos el ejemplo (3), que ofrecemos ahora con un contexto más amplio.

(10) P: [...] /total que/ el otro niño vino $\uparrow /$ le tomaron la temperatura $\uparrow$ tenía fiebre y no le pudieron operar/ así que pasó él el primero// pasó él y nos dijo el cirujano/ no os asustéis/ va a llorar// porque va a llorar $\downarrow$ - cuan - cuando se lo llevaron $\uparrow$ no/ porque empezaron a gastarle bromas $\uparrow /$ (Conversación [G.68.B.J+G.69.A.J], Grupo Val.Es.Co 1995: 201)

El contexto del ejemplo, como se señaló anteriormente en la nota 3, lo constituye una conversación coloquial prototípica en que el hablante $\mathrm{P}$, mujer, de más de 55 años y nivel de instrucción bajo, reproduce las palabras de otro al relatar una situación anterior, es decir, trae a la conversación un discurso referido. Se trata del intercambio comunicativo entre el cirujano que va a operar a un niño (intervención quirúrgica menor) y su madre, conversación a la que asiste P. En la transcripción del grupo Val.Es.Co, P está reproduciendo en el transcurso de una conversación posterior las palabras del cirujano pero con su propia formulación, y en este contexto profiere el enunciado (10). En (10) observamos varias cosas:

1. Existencia de dos miembros $p$ (antecedente) y $q$ (consecuente) con estructura sintáctica externa y formalmente causal. El molde sintáctico es el de la subordinación causal: $p$, porque $q$.

2. Pausa marcada entre $p$ y $q$ y entonación en cadencia al final del grupo.

3. Estructura de naturaleza tautológica. Repetición, aunque no necesariamente en sus propios términos, de $p$ cuando porque introduce $q$.

4. El enunciado es asertivo y el segundo miembro no puede orientarse de forma contraria al primero (si el primero es afirmativo, el segundo también lo es; si el primero es negativo el segundo también).

5. El orden es fijo. No hay posibilidad de anteposición de $q$ a $p$.

A partir de ahora, nos referiremos a estas construcciones con la denominación provisional de 'causales evidenciales tautológicas'. En ellas, la pausa marcada entre los dos miembros conectados por porque indica que se trata de una causal periférica y explicativa de carácter epistémico, no hay, pues, integración de $q$ en el sintagma verbal de la principal. La repetición de lo expresado en $p$, resulta de baja informatividad (la baja informatividad es propia de la oralidad), lo que refuerza el carácter epistémico de $q$, por el cual el hablante presenta su opinión sobre lo que va a suceder evaluando lo expresado en $p$ como indudable, es decir, $q$ se convierte en un refuerzo argumentativo fuerte de $p$ y por eso también adquiere valor confirmativo respecto de la aserción anterior. La naturaleza tautológica de la estructura guía la inferencia hacia la evidencialidad con confirmación 
fuerte, implicando que 'es completamente seguro lo que se ha expresado en $p$ y lo es gracias al compromiso del hablante que asume la veracidad del cumplimiento del acto futuro'. La pausa marcada entre los dos miembros del enunciado indica nuevamente este compromiso. El tonema final en cadencia de todo el grupo aumenta la seguridad de la aserción. La repetición tautológica convierte en foco lo expresado en $q$; a ello contribuye también el orden fijo de la secuencia. Porque presenta el miembro que introduce como justificación de lo afirmado en el primer aserto, en la órbita, pues, de las causales explicativas. Pero apunta a una justificación no expresa, sino deducida a partir de la seguridad con la que el hablante manifiesta lo que dice. La reconstrucción del proceso argumentativo podría ser la que sigue: 'si se afirma que el niño va a llorar es porque el hablante que emite el enunciado tiene razones sólidas para ello que, en este caso, se fundamentan en la experiencia que tiene como cirujano que ha operado a muchos niños', es decir, el argumento fuerte introducido por porque se basa en la competencia del hablante, que se presenta, a través de su aserción, como autorizado para decir lo que dice. De este modo, el hablante se compromete con lo dicho y con lo comunicado a través del encadenamiento de dos actos de habla consecutivos: uno asertivo explícito (va a llorar) y otro, si la orientación del acto es futura, compromisivo pero inferido (te aseguro que va a llorar), activado este último por medio de porque, que se comporta como un indicador de la fuerza ilocutiva compromisiva con el apoyo, como ya se ha señalado, de la pausa marcada, la repetición del primer argumento asertivo y el tonema final descendente. Esto da como resultado un enunciado pragmático que tiende a bloquear las posibles réplicas, si las hubiera en el intercambio comunicativo, puesto que inhibe un posible desacuerdo del interlocutor, que tendría que argumentar contra la afirmación fuerte (aunque hipotética) del hecho introducido por porque.

Un uso similar al que acabamos de exponer para el español le resulta sorprendente a Wüest para el francés. Al referirse a los valores epistémicos ('argumentativos' los denomina él frente a 'causales') de la conjunción parce que, que explica dentro de la teoría de los topoï, comenta lo siguiente: "s'il ne s'agit pas d'un simple jeu des mots", a propósito de este ejemplo de Marrou: 
(11) Louis XIV, lui, qui avait du goût parce qu' il avait un goût (Wüest 2012: 92, nota 8) ${ }^{17}$

Sin embargo, al analizar los "emplois particulier de car" (2012: 102) encuentra otros usos a los que llama "argumentations tautologiques" y cuya estructura en espejo sí que se considera como vehículo de una causal argumentativa. Lo vemos en el ejemplo, nuevamente de Marrou correspondiente a un ensayo:

(12) un mythe, car c' est un mythe,

también en el ejemplo de la obra teatral Les Justes de Camus:

(13) Stepan. -J'ai eu honte de moi-même, une seule fois, et par la faute des autres. Quand on m'a donné le fouet. Car on m'a donné le fouet.

Y por último, en la novela de Butor, La modification:

(14) $[\ldots]$ Il faudrait bien un jour qu'elle sache... Car il le faudra bien, n'est-ce pas?

Las causales evidenciales tautológicas aparecen fundamentalmente en las conversaciones coloquiales prototípicas (v. ej. 10):

(15) Enfrente del mercado de San Miguel está la Panasonic y tienen todos los recambios desde el primer modelo hasta el último [de aspirador], porque los tienen. (Conversación coloquial. Fragmento monológico que trae al momento de enunciación el relato oral de una conversación precedente. Informante varón, de 70 años y formación superior, variedad castellano central con influencia del catalán,18/11/2013)

(16) Como no tienen cánticos para animar a su equipo[el Real Madrid], porque no los tienen... (turno en una conversación coloquial; hablante varón, 18 años, estudiante universitario, variedadcastellano-central, 7/I/2014)

Pero también podemos encontrarlas en conversaciones coloquiales periféricas con distinto grado de formalidad, como sucede en este ejemplo, correspondiente a una conferencia:

(17) Pero la necesidad de que exista un tribunal, organismo especializado en la lucha contra la corrupción, es absolutamente necesario. Porque si ahora, en la propia Audiencia Nacional, que es un tribunal especializado y que tiene medios, porque tiene medios, hay procesos que duran 6-7 años [...].(Ángel Juanes, presidente de la Audiencia Nacional, Ponencia en las Jornadas sobre la situación de la Justicia, celebradas en la Asamblea de Madrid, 30 de septiembre de 2013. Difusión radiofónica a través de la cadena SER. Texto de oralidad secundaria)

o en registros escritos, pero cercanos a la oralidad como en el siguiente correo electrónico:

(18) Va a aparecer porque va a aparecer (correo electrónico privado entre dos colegas, a propósito del extravío por mensajería de un manuscrito, 26 de abril de 2012)

\footnotetext{
${ }^{17}$ El trabajo de Wüest se basa en un corpus de textos escritos por lo que no nos ofrece información, más allá de las pausas que se corresponden con signos de puntuación escritos, sobre la entonación y empleo de las pausas en las ocurrencias analizadas.
} 
Tanto en la oralidad primaria como secundaria o en registros escritos coloquiales, la tautología no tiene que formularse estrictamente en espejo, sino que puede haber variaciones, siempre que se mantengan expresos los elementos fundamentales de la primera aserción, como comprobamos en

(19) Yo creo que a Rajoy no le puede gustar, porque no le gusta, que ningún ministro esté en el centro de las declaraciones. (Carmen Morodo, periodista de La Razón. Cadena SER, $8: 18$ hs., $13 / 11 / 2013$ )

con variación formal sobre el esquema tautológico que lo sitúa a medio camino entre el valor de causal explicativa propia (base para afirmar lo que se dice por la observación de hechos repetidos o frecuentes, siempre desde la perspectiva del enunciador, Carmen Morodo) y el valor epistémico evidencial. Es probable que contextos como este constituyan el paso del valor causal al evidencial.

Otro ejemplo de variación sobre el esquema tautológico lo tenemos en

(20) Una persona sin espíritu, porque se la ve sin espíritu (turno de palabra en una conversación coloquial; hablante varón, 52 años, formación superior, variedad castellanocentral, 30/XII/2013)

en que, más que el compromiso con lo dicho, pues no se trata de un acto proyectado hacia el futuro, el enunciador presenta el miembro introducido mediante porque como refuerzo de la aserción precedente.

El hecho de que las causales tautológicas de este tipo aparezcan fundamentalmente en entornos conversacionales lleva a que puedan convertirse en un recurso para recrear la oralidad, como sucede en guiones de cine, televisión o en obras teatrales:

(21) [Refiriéndose a su marido] Que me acuse de infidelidad cuando no tiene pruebas, porque no tiene pruebas. (Valeria, personaje de la serie Amar es para siempre, Antena 3, 16/XII/13, 17:05 hs.)

$\mathrm{Y}$, aunque no son frecuentes estas construcciones en textos escritos más formales o elaborados, sí podemos encontrar causales epistémicas que pueden verse situadas a la izquierda de los porques evidenciales, entre las causales explicativas propias y las epistémicas, dentro de la organización gradual de la causalidad, como en esta interesante aparición de porque en estilo indirecto:

(22) Siempre le había achacado falta de prudencia, una virtud que él había convertido en su norma de vida, pero ahora descubría en su retoño cualidades de valentía, inteligencia, agilidad, y también sagacidad y astucia. Dichas cualidades habían salvado a la monarquía de un golpe mortal y estaba seguro de que le servirían para protegerse de las próximas 
intentonas de esos aventureros. Porque habría otras, de eso estaba seguro. (Moro, Javier [2011]: El imperio eres tú, Barcelona, Planeta, pág. 171. ESPAÑA).

\subsection{Las causales tautológicas y el bloqueo de la réplica}

Si bien hemos llamado 'causales tautológicas' a estas construcciones de valor pragmático evidencial, existen otro tipo de causales tautológicas relacionadas con el bloqueo de las réplicas del tipo

\section{(23) Porque sí / Porque no}

Nos referimos a construcciones en las que el hablante no desea dar explicaciones sobre la motivación de sus actos y, a partir de un esquema causal puro en el que la causa siempre aparece como no consabida, el hablante manifiesta su deseo de no justificarse ante el oyente, bien porque no quiere hacerlo o porque carece de la información suficiente para ello (v. infra, ej. 26).

(24) Lo hice porque lo hice

(25) Aquí vine porque vine

A la feria de las flores (La feria de las flores, canción de Jorge Negrete)

En estos casos, a diferencia de lo que sucedía en las construcciones evidenciales tautológicas, no hay pausa obligatoria entre los dos miembros del enunciado, lo que integraría el miembro introducido mediante porque en el predicado de la oración precedente. Y también a diferencia de las causales evidenciales tautológicas suelen funcionar como respuesta a preguntas o peticiones de información:

(26) - ¿Entonces él quemó la pintura de Rembrandt?

-Sí, eso me dijo mi madre.

-¿Pensando que era de Rembrandt y valía muchísimo?

-Era de Rembrandt y valía mucho -ratificó el médico, incapaz de entender las entretelas de aquellas preguntas o pensando que su interrogador del momento sufría de endurecimiento de la corteza cerebral provocada por grave infección urinaria.

- ¿Cómo él lo sabía? -insistió Conde.

- ¡Lo sabía porque lo sabía, digo yo...! Era un retrato de un judío que se parecía a Cristo. Él lo había visto muchas veces en su casa, en Cracovia. (Padura, Leonardo [2013], Herejes, Barcelona, Tusquets Editores, pág. 188-189. CUBA)

Así consideradas, este tipo de causales puras tautológicas puede convertirse en un recurso oral para suplir las dificultades a la hora de formular de forma precisa la causa objeto de la petición informativa, lo que se observa en el ejemplo (27), con alargamiento vocálico final de porque, propio de las pausas de planificación: 
(27) No se respeta porqueeee [...] no se respeta. (Telediario Telecinco, $1^{\text {a }}$ edición. 10/I/2014)

El ejemplo corresponde a una entrevista a pie de calle, en Sevilla. En ella se pregunta al entrevistado ocasional por el límite de velocidad en España. El entrevistado es un varón de unos 50 años, parece tener formación, al menos, media y presenta una variedad meridional fonéticamente muy atenuada, con nivelación de los rasgos más prototípicamente meridionales. Lo imprevisto de la situación parece forzar el uso de esta causal tautológica como procedimiento de construcción y cierre del enunciado.

La vinculación de estas construcciones también tautológicas con las causales puras se comprueba en el hecho de que en determinados contextos puede existir ambigüedad entre los dos tipos de construcciones, o incluso pueden interpretarse los esquemas tautológicos con valor de bloqueo de la réplica como verdaderas causales puras, pues se ajustan al motivo que el enunciador presenta como tal cuando responde a una pregunta o a una petición de información, como sucede en

(28) Queremos dos cosas - dijo Antonio con cansancio, como si llevase noches y noches sin dormir en su enorme apartamento minimalista, ni probablemente en ningún hotel, sino en el sofá de algún amigo-. Una que nos devuelvas lo que te llevaste. Dos: que nos digas dónde está Irina.

Manuela intervino justificándose, pidiéndome ayuda, aunque nadie le estuviese preguntando.

-Les he dicho que no lo sé porque no lo sé.

-Yo tampoco lo sé -dije-. No lo sabe nadie. (Clara Sánchez [2013], El cielo ha vuelto, Barcelona, Planeta, pág. 367.ESPAÑA)

Aunque este tipo de causales tautológicas comparte con las causales evidenciales tautológicas el debilitamiento del valor causal prototípico (a pesar de la forma sintáctica externa); también el hecho de que el segundo miembro no pueda orientarse de forma contraria al primero (si el primero es afirmativo, el segundo también lo es; si el primero es negativo el segundo también) y, finalmente, el mantenimiento de un orden fijo, con imposibilidad de anteposición de $q$ a $p$, frente a las causales evidenciales tautológicas, las causales tautológicas, a las que llamaremos provisionalmente, 'de bloqueo de la réplica' se caracterizan por:

1. Dentro de la causalidad, se sitúan más cercade las causales puras.

2. No hay pausa obligatoria entre los dos miembros del enunciado (pporqueq), por tanto, hay integración del miembro introducido por porque en el predicado de la oración precedente. Se situarían al lado de las causales integradas. 
3. Suelen aparecer en contextos reactivos (respuestas a preguntas o peticiones de información, implícitas o no), en que la baja informatividad ${ }^{18}$ les confiere la capacidad de funcionar como bloqueo de la réplica.

4. Estructura tautológica en espejo. No admiten variaciones en el esquema tautológico: $p$ debe ser repetido como $q$, en sus propios términos.

Este tipo de construcciones, también tautológicas, pero no evidenciales, pueden interpretarse como explicaturas (Sperber y Wilson 1986, 1995: 182). En la TR (teoría de la relevancia), los enunciados pragmáticos necesitan de un enriquecimiento contextual para ser interpretados. Esto es lo que perciben los hablantes: "sentidos contextualmente enriquecidos, ya como explicaturas, ya como implicaturas" (Portolés 2004: 148). En la TR el significado codificado, indeterminado siempre, constituye una plantilla semántica, sobre la que el hablante construye un enunciado explícito que puede ser interpretado en dos niveles, el de la explicatura, cuando las distintas posibilidades interpretativas dependen de la forma lingüística codificada en relación con el contexto. En este caso, todas las posibilidades están explícitas en la proposición y es la relación con el contexto (entorno cognitivo) la que decidirá cuál es la que más conviene. En el caso de la implicatura la suposición comunicada no es explícita, sino implícita. En estas estrategias para un posible bloqueo de la réplica a través de construcciones causales tautológicas, lo explicitado necesita de un proceso de desambiguación para seleccionar cuál de las posibilidades tiene la pertinencia óptima en ese contexto, la interpretación como causal pura o como causal tautológica $^{19}$.

\subsection{Los marcadores de evidencialidad y porque}

No pueden explicarse adecuadamente las construcciones evidenciales tautológicas con porque sin ponerlas en relación con otros marcadores evidenciales. Desde una perspectiva formal, nos hemos acercado a porque como un nexo conjuntivo. Así lo aconsejan su carácter proclítico, su integración en la estructura oracional y su escaso empleo como

\footnotetext{
${ }^{18}$ Cuando hablamos de "baja informatividad" nos referimos al hecho de que no hey aporte de nuevos contenidos en el miembro introducido por porque, desde el punto de vista del significado literal.

${ }^{19}$ Carston (2012)ha revisado en diversas publicaciones el concepto de explicatura. Es muy interesante la relación que establece entre lo lingüísticamente codificado y los significados ad hoc que subyacen a numerosos enunciados metafóricos y que permiten explicar no solo las metáforas, sin otro tipo de usos o literales del lenguaje. Para este autor: "The key claim for present purposes is that metaphorically used words and phrases are cases of pragmatic broadening of the linguistically encoded concepts and so, like other loose uses, their interpretation results in an ad hoc concept which is a component of the speaker's explicature." (Carston 2012: 478).
} 
marcador discursivo, pues no suele aparecer autónomamente (sin establecer conexión oracional), a diferencia de lo que sucede con los usos consecutivos de pues, los ilativocausales de que o los contraargumentativos de pero. Sin embargo, los valores pragmáticos de la construcción evidencial con porque no pueden ser explicados sin tener en cuenta otra clase de elementos: los marcadores del discurso o partículas discursivas, denominación que va ganando terreno en la actualidad. Siguiendo la definición del profesor Portolés (1993, 1998: 25-26 y 2004: 282), entendemos los marcadores discursivos como unidades lingüísticas invariables, que no ejercen función sintáctica dentro de la oración, cuyo cometido común consiste en guiar las inferencias que tienen lugar en la comunicación (significado procedimental). El tipo de orientación inferencial dependerá de las propiedades morfosintácticas, semánticas y pragmáticas de cada marcador o cada grupo de marcadores ${ }^{20}$. Muchos lingüistas han señalado el empleo de muchas conjunciones más allá de la oración ${ }^{21}$.En el caso del porquede las construcciones evidenciales tautológicas, la estructura sintáctica es superficialmente causal (porque establece una conexión entre dos oraciones), pero comparte con las partículas, además de la invariabilidad, por otro lado intrínseca a las conjunciones, la orientación inferencial en un sentido determinado. Esta orientación inferencial convierte este empleo discursivo de porque en una estrategia comunicativa para transformar el argumento introducido en un refuerzo fuerte de la aserción anterior, pues señala algo como indudable o evidente ${ }^{22}$. Este contexto de porque puede alternar con el empleo de marcadores de evidencia como desde luego, naturalmente, por supuesto y claro $^{23}$, todos ellos pertenecientes a la categoría general de la modalidad epistémica, que tiene como características principales la aparición en enunciados declarativos y la introducción de aserciones que reflejan "cómo enfoca al hablante el mensaje que el marcador introduce" (Martín Zorraquino y Portolés Lázaro 1999: 4146). Ambas características también se dan en los construcciones de porque analizadas. Además, los marcadores de evidencia, incluidos por Martín Zorraquino y

\footnotetext{
${ }^{20}$ Aunque hay aspectos, como su descripción morfológica (Martín Zorraquino 1998, 2010)todavía bastante discutidos, a la hora de caracterizar los marcadores del discurso como clases de palabras.

${ }^{21}$ Martín Zorraquino (2010: 113-120) hace una presentación general de las conjunciones como marcadores discursivos en su trabajo "Los marcadores del discurso y su morfología".

${ }^{22}$ El tipo de porque analizado comparte, además, con la clase de los marcadores el hecho de no poder ser negado y su capacidad focalizadora.

${ }^{23}$ Para una análisis más detallado sobre estos marcadores evidenciales pueden consultarse los trabajos de Freites Barros (2006), Fuentes Rodríguez (1993), González Pérez (2012), Martín Zorraquino (2011) y Sánchez Jiménez(2008).
} 
Portolés Lázaro (1999) dentro de los marcadores conversacionales, constituyen pragmáticamente un refuerzo de la aserción que se efectúa sobre el miembro al que afectan, pues son operadores discursivos y en el caso de porque, sobre el miembro precedente, pues realiza un tipo de conexión:

(29a) Va a llorar, porque va a llorar

(29b) (Va a llorar) Desde luego que va a llorar

(29c) (Va a llorar) Por supuesto que va a llorar

(29d) (Va a llorar) Naturalmenteque va a llorar

(29e) (Va a llorar) Claro que va a llorar.

Aunque porque puede alternar con los marcadores evidenciales anteriores, no puede hacerlo en cualquier contexto ni libremente. La aparición del porque evidencial exige la presencia de una miembro precedente que se repite para focalizar sobre él la atención; pues, evidentemente, porque no es un marcador discursivo, aunque dé lugar a construcciones que activan valores pragmáticos específicos en determinados contextos. Pero lo que separa las causales evidenciales tautológicas de los marcadores de evidencialidad son los efectos contextuales que activan cada uno de ellos. Solamente el entorno porque(en construcción tautológica) es capaz de marcar el compromiso patente del hablante con lo expresado en la segunda oración, relacionando dos actos de habla, uno asertivo y otro compromisivo implícito (cuando el segundo acto de habla se proyecta hacia el futuro), mediante los cuales el enunciador es el responsable, la garantía del cumplimiento del contenido proposicional del enunciado.

\section{Conclusiones}

En este trabajo hemos analizado valores pragmáticos de la causalidad que no han sido objeto de estudio hasta el momento. Esta toma de contacto con los fenómenos estudiados se ha abordado partiendo de los entornos discursivos en que, sin dejar de establecer externamente un tipo de conexión oracional, porque ha debilitado los valores causales para cargarse de otros valores pragmáticos. El estudio de estos entornos resulta interesante para un mejor conocimiento de la fijación del significado procedimental. En el caso de porque, no hay transformación en partícula discursiva, ni siquiera en coexistencia con los valores causales prototípicos. Pero sí existen marcas que activan determinados efectos contextuales: 
1. Existencia de estructura tautológica.

2. Orden fijo de los dos miembros que conecta porque, lo que impide la anteposición del segundo miembro.

3. Coorientación de los argumentos enlazados mediante porque.

El análisis de los casos estudiados revela también la existencia de, al menos, dos tipos de valores en las construcciones causales tautológicas: los evidenciales y los de bloqueo de la réplica. Los dos tipos se explican teniendo en cuenta la causalidad como un fenómeno gradual desde la causa objetiva a valores cada vez más subjetivos:

1. Causal pura

2. Causal justificativa

3. Causal epistémica.

4.1. Causales tautológicas evidenciales

4.2. Causales tautológicas de bloqueo de la réplica.

El grado 4 en la causalidad (anulación de los valores causales prototípicos) y en el avance de la pragmatización de los usos discursivos de porque, estaría constituido por las construcciones tautológicas estudiadas, que se situarían en distintos lugares: con las causales epistémicas los valores evidenciales de porque y con las causales puras los valores de bloqueo de la réplica. El carácter de causal periférica del tipo 4.1. frente al de causal integrada del tipo 4.2. avala este análisis. El tipo de inferencia de las dos construcciones causales tautológicas también refuerza esta interpretación; las causales evidenciales se explican en el nivel de la implicatura, activando supuestos compartidos o enunciaciones anteriores, frente a las de bloqueo de la réplica, que pueden constituir un tipo de explicatura y dar lugar a procesos de desambiguación para suprimir la posibilidad de interpretación como causal pura.

Somos conscientes de que un estudio más profundo de estas construcciones con porque exigiría la comparación con los de otros nexos causales, especialmente con que, la elaboración de un corpus de rastreo del fenómeno y el contraste con otras estructuras tautológicas (Es alto, pero alto / Un mercedes, es un mercedes[tipo de coche, prototípicamente caro y de gran calidad]). 
Bibliografía

Bello, A. ([1847] 1998): Gramática de la lengua castellana destinada al uso de los americanos. Con las notas de R. J. Cuervo y R. Trujillo (eds.). Madrid: Arco Libros, 2 vols.

Bosque, I. y V. Demonte (1999) (eds.): Gramática descriptiva de la lengua española. Vol. III. Madrid: Espasa Calpe.

Briz Gómez, A. (2011): Lo discursivo de las partículas discursivas en el Diccionario de partículas discursivas del español (DPDE). En: Aschenberg, H. y Ó. Loureda Lamas (eds.), Marcadores del discurso. De la descripción a la definición.Fráncfort del Meno: Iberoamericana/Vervuert, 77-108.

Briz Gómez, A. y otros (eds.)(2008): Diccionario de partículas discursivas del español. DPDE. < http://www.dpde.es >

Carston, R. (2012): Metaphor and the literal/non-literal distinction. En: Allan, K. y K. M. Jaszczolt (eds.), The Cambridge Handbook of Pragmatics. Cambridge: CUP.

Freites Barros, F. (2006): El marcador de discurso Claro: funcionamiento pragmático, metadiscursivo y organizador de la estructura temática.Verba 33, 261-279.

Fuentes Rodríguez, C. (1993): Desde luego, Por supuesto, Naturalmente. Sociolingüística andaluza 8, 127-159.

Fuentes Rodríguez, C. (2009): Diccionario de conectores y operadores del español. Madrid: Arco Libros.

Galán Rodríguez, C. (1999): La subordinación causal y final. En: Bosque y Demonte (eds.), 3597-3642.

González Pérez, R. (2012): Sobre la historia de algunos marcadores confirmativos en español: la creación de por supuesto y su contraste con desde luego. En: Negroni, M. (ed.), Actas del II Coloquio Internacional Marcadores del discurso en lenguas románicas: un enfoque contrastivo. Vol. I. Buenos Aires: Ed. de la Facultad de Filosofía y Letras de la Universidad de Buenos Aires, 89-101. 
Grice, H. P. (1991 [en inglés 1975]): Lógica y conversación. En: Valdés Villanueva, L. M. (ed.), La búsqueda del significado. Lecturas de filosofía del lenguaje. Madrid: Tecnos, 22-40.

Groupe $\lambda$-1 (logique et langage) (1975): Car, parce que, puisque. Revue romane 10, 248-280.

Grupo Val.Es.Co. y A. Briz (eds.) (1995): La conversación coloquial. (Materiales para su estudio). Valencia: Universitat de València.

Grupo Val.Es.Co. y A. Briz (eds.)(2002): Corpus de conversaciones coloquiales. Madrid: Arco Libros.

Herrero Ruiz de Loizaga, F. J. (2005): Sintaxis histórica de la oración compuesta en español. Madrid: Gredos.

Lapesa, R. (1978): Sobre dos tipos de subordinación causal. En:Estudios ofrecidos a Emilio Alarcos Llorach.Vol. III. Oviedo: Universidad de Oviedo, 173-205.

Marcos Marín, F. (1979): A propósito de las oraciones causales. Observaciones críticas. Cuadernos de Filología. Studia Lingüística Hispanica II (1), 163-171.

Martín Zorraquino, Mª A. (1998): Los marcadores del discurso desde el punto de vista gramatical. En: Martín Zorraquino, $\mathrm{M}^{\mathrm{a}}$ A. y E. Montolío Durán (eds.), Los marcadores del discurso. Teoría y análisis. Madrid: Arco Libros, 19-53.

Martín Zorraquino, $\mathrm{M}^{\mathrm{a}}$ A. (2010): Los marcadores del discurso y su morfología. En: Loureda Lamas, Ó. y E. Acín Villa (eds.), Los estudios sobre marcadores del discurso en español, hoy. Madrid: Arco Libros, 93-181.

Martín Zorraquino, Mª A. (2011): De nuevo sobre la gramaticalización de desde luego. Lorenzo Hervás. Documentos de trabajo de Lingüística teórica y general20 (15), 365-378.

Martín Zorraquino, Mª A. y J. Portolés Lázaro (1999): Los marcadores del discurso. En: Bosque y Demonte (eds.),4051-4213.

Montolío, E. (1999): Las construcciones condicionales. En: Bosque y Demonte (eds.), 3643-3730. 
Pander Maat, H. y L. Degand (2001): Scaling causal relations and connectives in terms of Speakers Involvement. Cognitive Linguistics 12 (3), 212-245.

Pit, M. (2003): How to Express Yourself Whith a Causal Connective. Subjectivity and Causal Connectives in Dutch, German and French, Amsterdam/Nueva York: Rodopi.

Portolés Lázaro, J. (1993): La distinción entre los conectores y otros marcadores del discurso. Verba 20, 141-170.

Portolés Lázaro, J. (1998): Marcadores del discurso. Barcelona: Ariel.

Portolés Lázaro, J. (2004): Pragmática para hispanistas. Madrid: Síntesis.

Sánchez Jiménez, S. U. (2008): La creación de un marcador del discurso: naturalmente. En: Elvira, J. y otros (eds.), Lenguas, reinos y dialectos en la Edad Media ibérica. La construcción de la identidad.Madrid/Fráncfort del Meno: Iberoamericana/ Vervuert, 435-468.

Santos Río, L. (2003): Diccionario de partículas. Salamanca: Luso-Española de Ediciones.

Sperber, D. y D. Wilson (1994 [1986]): La relevancia. Comunicación y procesos cognitivos. Madrid: Visor.

Sweetser, E. (1990): From Etymology to Pragmatics. Cambridge: CUP.

Wilson, D. y D. Sperber (2004): Relevance Theory. En: Horn, L. R. y G. L. Ward (eds.), The Handbook of Pragmatics. Malden: Blackwell, 607-632.

Wüest, J. (2012): Argumentation et causalité. À propos des connecteurs parce que, car, et puisque.Vox romanica $71,77-108$.

Zufferey, S. (2012): Car, parce que, puisque revisited: Three empirical studies on French causal connectives.Journal of Pragmatics 44, 138-153.

Recibido: 29 de julio de 2014 Aceptado: 12 de noviembre de 2014 Revisado: 19 de diciembre de 2014

Publicado: 22 de junio de 2015 Actualizado: 3 de agosto de 2015 